\title{
SUPPORT FUNCTIONALS AND THEIR RELATION TO THE RADON-NIKODYM PROPERTY
}

\author{
I. SADEQI \\ Received 26 May 2002
}

In this paper, we examine the Radon-Nikodym property and its relation to the Bishop-Phelps theorem for complex Banach spaces. We also show that the Radon-Nikodym property implies the Bishop-Phelps property in the complex case.

2000 Mathematics Subject Classification: 46E99, 46J10.

1. Introduction. Let $X$ be a complex Banach space and let $C$ be a closed convex subset of $X$. The set of support points of $C$, written as $\operatorname{supp} C$, is the collection of all points $z \in C$ for which there exists nontrivial $f \in X^{*}$ such that $\sup _{x \in C}|f(x)|=|f(z)|$. Such an $f$ is called support functional. The point $z \in \operatorname{supp} C$ is called a strongly exposed point of $C$ if for all sequences $\left\{z_{n}\right\} \subset C$, $\lim _{n \rightarrow \infty}\left(\operatorname{Re} f\left(z_{n}\right)\right)=\sup _{C} \operatorname{Re}(f) \operatorname{implies}$ that $z_{n} \rightarrow z$, where Re denotes the real part.

In this paper, we will show that the unit ball of an infinite-dimensional function algebra has no strongly exposed points. Lomonosov [4] constructed a closed, bounded, and convex subset $C$ of a complex Banach space such that the set of support points of $C$ is empty. This means that the Bishop-Phelps theorem fails to hold in the complex case. We show below that for Hardy spaces, the Bishop-Phelps theorem does hold.

Bourgain [1] proved that if $X$ is a real Banach space, then the Radon-Nikodym property (RNP) and the Bishop-Phelps property (BPP) are equivalent. The precise definitions are given below.

It is natural to ask whether this equivalence remains true in the complex case. In this paper, we show that, appropriately defined, it does indeed hold for complex Banach spaces. Recall that a Banach space $X$ is said to have the RNP, provided that for every measure space $(\Omega, \Sigma, \mu)$ with $\mu(\Omega)<\infty$, and every $\mu$-continuous measure $T: \Sigma \rightarrow X$ of finite variation, there exists a Bochner integrable function $f: \Omega \rightarrow X$ such that $T(E)=$ $\int_{E} f d \mu$ for every $E \in \Sigma$.

Let $X$ and $Y$ be Banach spaces and let $L(X, Y)$ be the Banach space of all bounded linear operators from $X$ into $Y$. Suppose that $T \in L(X, Y)$ and $C$ is a nonempty and bounded subset of $X$, then we define $\|T\|(C):=\sup \{\|T x\|: x \in C\}$. A Banach space $X$ is said to have the BPP if for any nonempty, bounded, and closed subset $C$ of $X$, any Banach space $Y$, and any $T \in L(X, Y)$, there is an approximating sequence $\left(T_{n}\right)$ in $L(X, Y)$ for which each $\left(T_{n}\right)$ achieves its max norm $\left\|\left(T_{n}\right)\right\|(C)$ on $C$.

A subset $C$ of a Banach space is called dentable if for every $\epsilon>0$, there exists an $x \in C$ such that $x \notin \overline{\mathrm{co}}(C-\mathbb{N}(x, \epsilon))$, where $\overline{\mathrm{co}}$ denotes "closed convex hull" and $\mathbb{N}(x, \epsilon)$ is the 
open $\epsilon$-neighborhood of $x$. In the final part of this paper, we discuss uncountability of the set of normalized support functionals, a question posed by L. Zajicek in 1999.

2. The RNP for complex Banach spaces. Bourgain proved in [1] that if the real Banach space $X$ has the RNP and if $C$ is a closed, convex, and bounded subset of $X$, then the set of support functionals that strongly expose some point of $C$ is dense in $X^{*}$. Suppose that the complex Banach space $X$ has the RNP and that $C$ is a bounded, closed, and convex subset of $X$. Put

$$
H:=\left\{e^{i \theta} x: 0 \leq \theta<2 \pi, x \in C\right\}
$$

Let $B$ denote the closed convex hull of $H$. Then, as cited above, the set of real parts of linear functionals which strongly expose some point of $B$ forms a dense subset of $X_{r}^{*}$. Here $X_{r}$ is the underlying real Banach space. By the standard isometry $f \rightarrow \operatorname{Re} f$ between $X^{*}$ and $X_{r}^{*}$, these are the real parts of a dense subset of $X^{*}$. The strongly exposed points of $B$ are contained in $H$ since $H$ is closed, so by the theorem of Phelps,

$$
\sup |f|(C)=\sup \operatorname{Re} f(H)
$$

and the support functionals are dense in the complex case. Therefore, using the two theorems of Phelps and Bourgain, the RNP implies the Bishop-Phelps theorem in the complex case. If we show that the RNP implies the BPP in the complex case, the BishopPhelps theorem clearly holds for complex Banach spaces with the RNP without recourse to the theorems of Bourgain [1] and Phelps [6].

DEFINITION 2.1. Let $B$ be a nonempty, bounded, closed, and convex subset of the complex Banach space $X$. Let $Y$ be a Banach space and $T \in L(X, Y)$. Say that $T$ is an $F$-strongly exposing operator for the set $B$ if there exists some point $x \in B$ depending on $T$ such that every sequence $\left(x_{n}\right) \subset B$ satisfying

$$
\sup \|T\|(B)=\lim _{n \rightarrow \infty}\left\|T\left(x_{n}\right)\right\|
$$

has a subsequence converging to $\alpha x$ for some complex number $\alpha$ with $|\alpha|=1$.

If $X$ is a real Banach space, then the definition of an $F$-strongly exposing operator becomes Bourgain's definition of an $R$-strongly exposing operator, as follows.

Let $B$ be a nonempty, bounded, closed, and convex subset of the complex Banach space $X$. Let $Y$ be a Banach space and $T \in L(X, Y)$. T is called an $R$-strongly exposing operator for the set $B$ if there exists some point $x \in B$ depending on $T$ such that every sequence $\left(x_{n}\right) \subset B$ satisfying (2.3) has a subsequence converging to $x$ or $-x$ [1].

THEOREM 2.2 (Bourgain). Let B be a nonempty, bounded, closed, and convex subset of the real Banach space $X$. Assume that every nonempty subset of $B$ is dentable. Then for any Banach space $Y$, the set of all $R$-strongly exposing operators $T \in L(X, Y)$ for the set $B$ is a dense subset of $L(X, Y)$.

In the following discussion, we show why we need the definition of an F-strongly exposing operator to prove Bourgain's theorem in the complex case. Let $X$ be a complex 
Banach space and let $C$ be a circled, bounded, closed, and convex subset of $X$ (i.e., $\alpha C \subseteq C$ if $|\alpha|=1)$. Suppose that $T$ achieves its max norm $\|T\|(C)$ on $C$. Then there exists $x_{0}$ in $C$ such that

$$
\sup \|T\|(C)=\left\|T\left(x_{0}\right)\right\| \text {. }
$$

Choose $\alpha_{n}=i$; it is clear that

$$
\lim _{n \rightarrow \infty}\left\|T\left(\alpha_{n} x_{0}\right)\right\|=\sup \|T\|(C),
$$

and $\left(\alpha_{n} x_{0}\right)$ has no subsequence converging to $x_{0}$ or $-x_{0}$. So the circled subsets of $X$ have no $R$-strongly exposing operator. Also let $X_{r}$ and $Y_{r}$ be the underlying real part of the complex Banach spaces $X$ and $Y$, respectively. Since $L\left(X_{r}, Y_{r}\right)$ is not isomorphic to $L(X, Y)$ in general, we cannot directly use Bourgain's definition in the complex case.

THEOREM 2.3. Let $X$ be a complex Banach space with the RNP. Then $X$ possesses the $B P P$.

Proof. Let $C$ be a nonempty, closed, convex, and bounded subset of $X$. Let $Y$ be a complex Banach space. We must show that for $T \in L(X, Y)$, there is an approximating sequence $\left(T_{n}\right)$ for which each $\left(T_{n}\right)$ achieves its max norm on $C$. For $n \in \mathbb{N}$, define

$$
K_{n}:=\left\{T \in L(X, Y): \exists \xi \geq 0, \exists t \in X, S(T, \xi) \subset \bigcup_{|\alpha|=1} \mathbb{N}\left(\alpha t, n^{-1}\right)\right\},
$$

where $S(T, \xi):=\{x \in C:\|T x\| \geq\|T\|(C)-\xi\}$. Since $X$ has the RNP, every nonempty and bounded subset of $X$ is dentable, so $K_{n}$ is dense in $L(x, y)$ (see [1]). If $T \in K_{n}$ for each $n \in \mathbb{N}$, then there exists a sequence $\left(x_{n}\right)$ in $C$ such that $\lim _{n \rightarrow \infty}\left\|T x_{n}\right\|=\|T\|(C)$. There exists also $t \in X$ such that $\left(x_{n}\right)$ is contained in $F:=\{\alpha t:|\alpha|=1\}$. It follows that $x_{n}=\alpha_{n} t$, and there is a subsequence of $\left(x_{n}\right)$ converging to $\alpha_{0} t$ for some $\alpha_{0}$ with $\left|\alpha_{0}\right|=1$. Therefore, $T$ is an $F$-strongly exposing operator and achieves its max norm on $C$ at $\alpha_{0} t$. It follows that $T$ is an $F$-strongly exposing operator if and only if $T \in K_{n}$ for every $n \in \mathbb{N}$. Put $K:=\bigcap_{n=1}^{\infty} K_{n}$. Since any $K_{n}$ is dense in $L(X, Y)$ if we show that any $K_{n}$ is open, we conclude that $K$ is dense in $L(X, Y)$. Let $T \in K_{n}$ and suppose that $F \in L(X, Y)$ with $\|F-T\| \leq \xi / 3$. It is easy to see that $S(F, \xi / 3) \subseteq S(T, \xi / 3)$, so $F \in K_{n}$ and $K_{n}$ is open in $L(X, Y)$. The fact that each $T \in K$ is an $F$-strongly exposing operator and achieves its max norm on $C$ completes the proof.

COROLlary 2.4. The RNP implies the Bishop-Phelps theorem in the complex case.

Proof. Since the RNP implies the BPP in the complex case, the proof is clear.

That the BPP implies the RNP can be easily checked through Bourgain's proof in [1], so we have the following result.

THEOREM 2.5. The RNP and the BPP are equivalent for complex Banach spaces.

We are ready to examine the RNP for some complex Banach spaces. Let $\tau:=\left\{e^{i \theta}\right.$ : $0 \leq \theta \leq \pi\}$ and $L^{1}(\tau)$ denote the summable complex functions on $\tau$. The following discussion shows that some subspaces of $L^{1}(\tau)$ have the RNP so that the Bishop-Phelps 
theorem is true for them. The complex Bishop-Phelps theorem is still open for $L^{1}$; however, we can verify this theorem for some subspaces of $L^{1}$. Let $C(\tau)$ be the set of all continuous functions on $\tau$ and let $C^{0}$ denote the functions in $C(\tau)$ which are analytic with mean value zero. It is well known that

$$
\left(C(\tau) / C^{0}\right)^{*} \approx H^{1}(\tau)
$$

Since $H^{1}(\tau)$ is a separable space with a predual, it has the RNP and, hence, the BishopPhelps theorem is true for $H^{1}(\tau) \subset L^{1}(\tau)$. Also, all $H^{p}(1 \leq p<\infty)$ are separable and have a predual. The following theorem is an immediate consequence of this discussion.

THEOREM 2.6. The Bishop-Phelps theorem is satisfied for the Hardy spaces $H^{p}(1 \leq$ $p<\infty)$ in the complex case.

As mentioned above, a separable dual Banach space has the RNP. Since $H^{\infty}$ is not separable, it is still unknown whether the Bishop-Phelps theorem is true for $H^{\infty}$. Hensgen (see [3]) proved that the unit ball of $H^{\infty}$ has no strongly exposed points. Therefore, $H^{\infty}$ is guaranteed to lack both the RNP and the BPP. In the following, we will show that the unit ball of an infinite-dimensional uniform algebra has no strongly exposed point. As a result, such spaces do not have the RNP. So it is natural to ask whether the Bishop-Phelps theorem holds for infinite-dimensional uniform algebras.

Let $X$ be a nonempty set and let $K$ be a normed linear algebra. We denote by $\ell^{\infty}(X, K)$ the normed linear algebra of all bounded mappings of $X$ into $K$ with pointwise addition and scalar multiplication and with the uniform norm. For a bounded mapping $f$, the uniform norm is defined by

$$
\|f\|_{\infty}=\sup \{\|f(x)\|: x \in X\}
$$

A uniform algebra of functions on $X$ is a subalgebra of the Banach algebra $\ell^{\infty}(X, F)$, where $F$ is either the real or complex numbers. Given a nonempty topological space $X, C(X, K)$ denotes the linear space of all continuous mappings of $X$ into $K$ with pointwise addition and scalar multiplication. When $X$ is compact, $C(X, K)$ is a closed linear subspace of $\ell^{\infty}(X, K)$, and in particular, $C(X, F)$ is a uniform algebra of functions. The notation $C(X, F)$ is abbreviated to $C(X)$.

LEMmA 2.7. Let $X$ be a compact Hausdorff space such that $C(X)$ is infinite-dimensional. Then the unit ball of $C(X)$ has no strongly exposed point.

Proof. If $X$ admits no Baire diffuse measure (a nonnegative measure $\mu$ on $X$ is said to be diffuse if $\mu(V)>0$ for every nonempty open subset $V$ of $X)$, then the unit ball of $C(X)$ contains no exposed point (see [5]). So the proof is clear in this case. Let $X$ admit a diffuse measure and suppose that $\lambda \in C(X)^{*}$ exposes $f \in U$, where $U$ is the unit ball of $C(X)$. Then $|f(t)|=1(t \in X)$. Since $X$ admits a diffuse measure and $\|f\|=1$, a result of Eberlein's guarantees that accumulation points can be approximated by sequences (see [7]). We can assume that there exists a sequence $\left(t_{n}\right) \subset X$ such that $f\left(t_{n}\right) \rightarrow 1$. Choose pairwise disjoint open sets $U_{n}$ in $X$ such that $t_{n} \in U_{n}$. Also choose $h_{n} \in C(X)$ with $0 \leq h_{n} \leq 1, h_{n}\left(t_{n}\right)=1$, and $h_{n}=0$ on $K \backslash U_{n}$. Put $g_{n}=1-h_{n}$; clearly $\left\|g_{n}\right\| \leq 1$ 
and $g_{n}(t) \rightarrow 1$ for each $t \in X$. Hence $g_{n} f(t) \rightarrow f(t), t \in X$. Also by the Hahn-Banach theorem, it is easy to see that $\lambda$ must be of the form

$$
\lambda(g)=\int_{X} g \bar{f} d \mu .
$$

It is clear that $\lambda\left(g_{n} f\right) \rightarrow \lambda(f)$, but $\left\|g_{n} f-f\right\|=\left\|h_{n} f\right\| \geq 1$. So $f$ cannot be a strongly exposed point. Also since any uniform algebra is a commutative $B^{*}$-algebra, by the Gelfand-Naimark theorem, $A$ is an isometric isomorphism of $C\left(\Delta_{A}\right)$, where $\Delta_{A}$ is the maximal ideal space of $A$. Therefore, as cited above, $A$ has no strongly exposed point, and we have the following result.

THEOREM 2.8. The unit ball of an infinite-dimensional $C^{*}$-algebra has no strongly exposed point.

It is well known that if $X$ is a complex Banach space with the RNP, then the set of support functionals that expose some points of the unit ball of $X$ is norm dense in $X^{*}[1]$.

COROLlary 2.9. An infinite-dimensonal separable $C^{*}$-algebra possesses neither the RNP nor the BPP; hence it has no predual.

\section{The set of normalized support functionals}

Problem 3.1. Suppose that $X$ is a real Banach space with $\operatorname{dim} X>1$ and $C \subseteq X$ is a bounded, closed, and convex subset of $X$. Is the set of normalized support functionals $\Sigma(C)$ an uncountable subset of $S_{X^{*}}$ ? (Due to L. Zajicek).

Phelps has made the following observations. Suppose that $\Sigma(C)$ is countable; then it has no interior point, so $S_{X^{*}} \backslash \Sigma(C)$ is a dense $G_{\delta}$ set in $S_{X^{*}}$. Since $\Sigma(C)$ is dense in $S_{X^{*}}$, from the Bishop-Phelps theorem, we conclude that $X^{*}$ is separable. If $X$ is reflexive, then any linear functional of $S_{X^{*}}$ supports $C$, which is a contradiction. Since $\Sigma(C)$ is countable, $X$ must be a nonreflexive space with a separable dual space. Also $C$ must have an empty interior because otherwise we may assume that $0 \in \operatorname{int} C$, and since $\operatorname{dim} X>1$, it is possible to have a two-dimensional subspace $M$ of $X$. So if $f \in S_{M^{*}}$, then it supports $M \cap C$. By the Hahn-Banach theorem, there is an extention $f^{\prime}$ of $f$ in $\Sigma(C)$. That is, there is one-to-one map from the uncountable set $\Sigma(M \cap C)$ into $\Sigma(C)$, which is impossible.

THEOREM 3.2. Let $X$ be a weakly sequentially complete (w.s.c.) Banach space and let $C$ be a closed, convex, and bounded subset of $X$. Then $\Sigma(C)$ is uncountable.

Proof. If $\Sigma(C)$ is countable, since $\Sigma(C)$ is dense in $S_{X^{*}}, S_{X^{*}}$ is separable. By Dounford-Pettis theorem (see [2]), $X^{*}$ possesses the RNP. Let $\left(x_{n}\right)$ be a bounded sequence in $X$. Then $Y$, the closed linear span of $\left(x_{n}\right)$, is a separable subspace of $X$. Since $X^{*}$ has the RNP, $Y^{*}$ is separable. By a classical result of Banach, $\left(x_{n}\right)$ has a weak Cauchy subsequence in $Y$ (again denoted by $\left(x_{n}\right)$ ), which is also a weak Cauchy sequence in $X$. Since $X$ is w.s.c., then $\left(x_{n}\right)$ is a weakly convergent sequence, therefore, any bounded sequence $\left(x_{n}\right)$ is a weakly convergent sequence in $X$, and so $X$ is a reflexive space. Thus $\Sigma(C)=S_{X^{*}}$, an uncountable set. 
REMARK 3.3. If any nonempty subset of a closed, convex, and bounded set $C$ is dentable, then by a result of Bourgain (see [6]), for such a set $C$, the set of support functionals is $G_{\sigma}$ dense in $X^{*}$. And by a classical theorem that the Banach space $X$ has no countable $G_{\sigma}$ dense subset, we conclude that $\Sigma(C)$ is uncountable.

Acknowledgment. My sincerest thanks go to Professor R. Phelps for his assistance.

\section{REFERENCES}

[1] J. Bourgain, On the dentability and the Bishop-Phelps property, Israel J. Math. 28 (1977), 263-271.

[2] J. Diestel, Geometry of Banach Spaces, Springer-Verlag, Berlin, 1975.

[3] W. Hensgen, Exposed points in Lebesgue-Bochner and Hardy-Bochner, J. Math. Anal. Appl. 198 (1996), 780-796.

[4] V. I. Lomonosov, A counterexample to the Bishop-Phelps theorem in complex spaces, Israel J. Math. 115 (2000), 25-28.

[5] R. R. Phelps, Extreme points in function algebras, Duke Math. J. 32 (1977), 267-278.

[6] _ The Bishop-Phelps theorem in complex spaces: an open problem, Pure Appl. Math. 131 (1991), 337-340.

[7] T. S. S. R. K. Rao, There are no denting points in the unit ball of $W C(K, X)$, Proc. Amer. Math. Soc. 127 (1999), no. 10, 2969-2973.

I. Sadeqi: Departement of Mathematics, Sahand University of Technology, P.O. Box 51335-1996, Tabriz, Iran

E-mail address: esadeqi@sut.ac.ir 


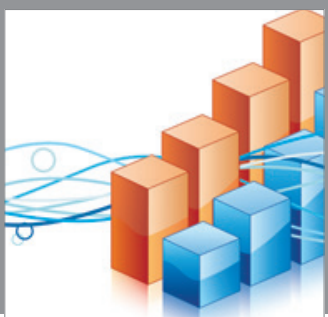

Advances in

Operations Research

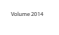

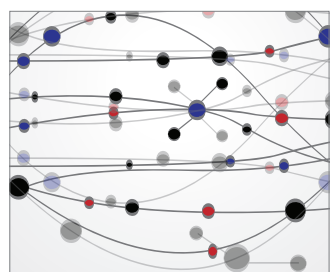

\section{The Scientific} World Journal
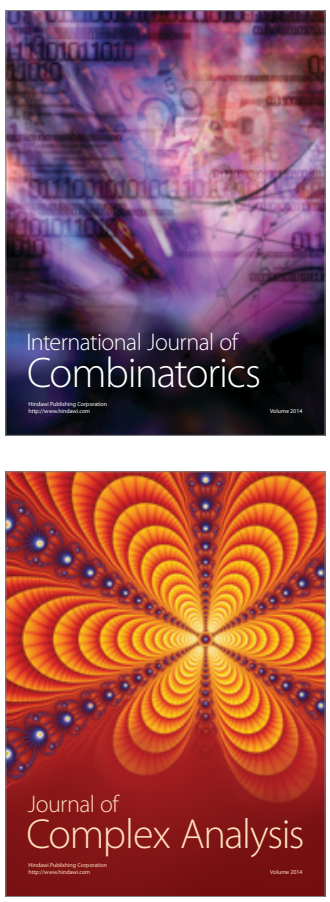

International Journal of

Mathematics and

Mathematical

Sciences
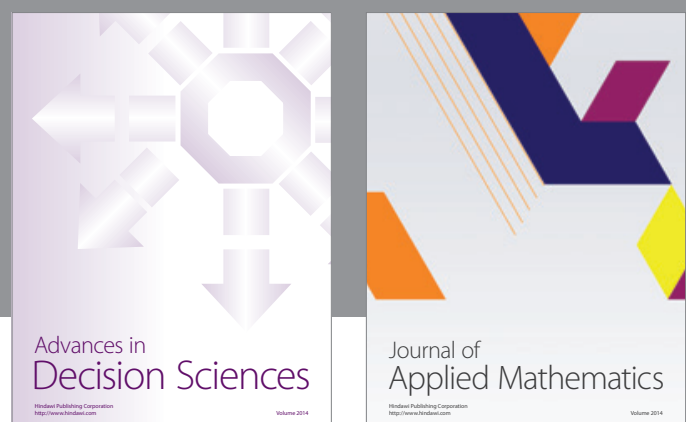

Journal of

Applied Mathematics
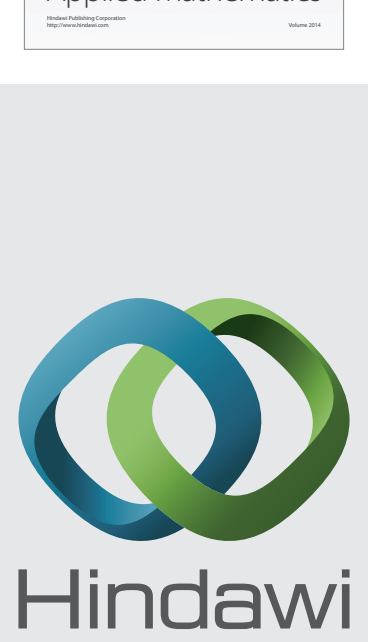

Submit your manuscripts at http://www.hindawi.com
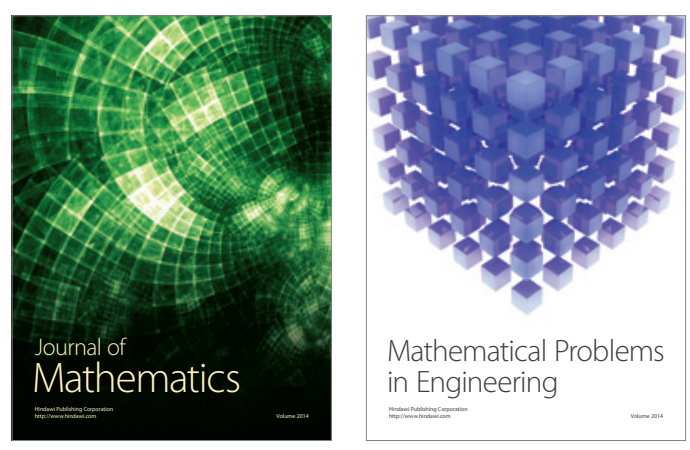

Mathematical Problems in Engineering
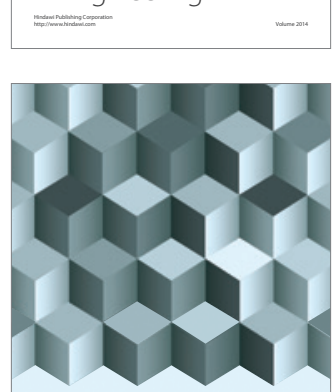

Journal of

Function Spaces
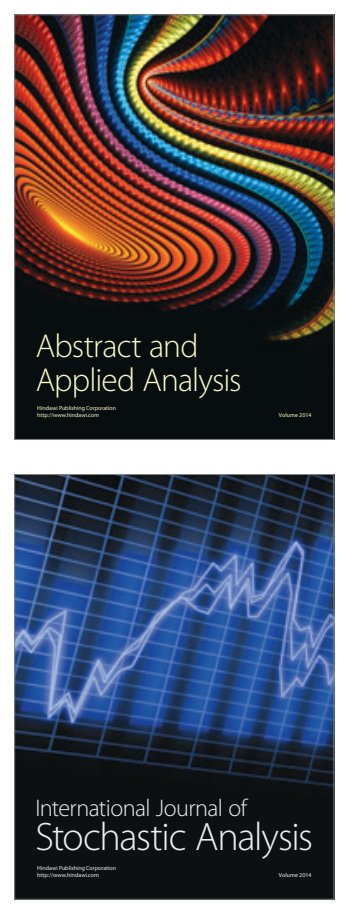

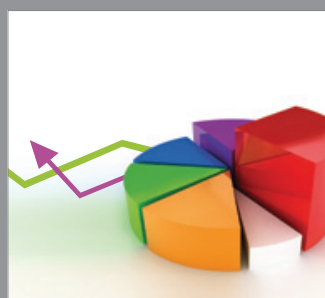

ournal of

Probability and Statistics

Promensencen
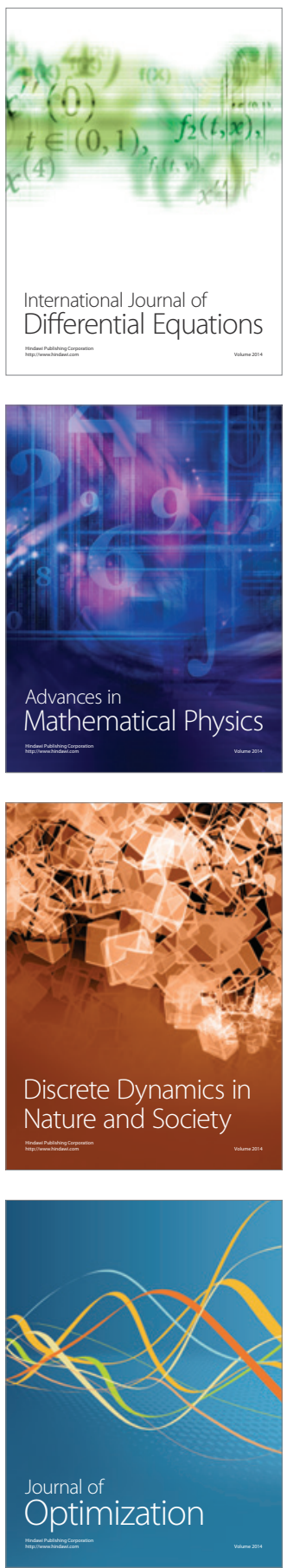